\title{
Criminologie
}

\section{Éditorial : homicide conjugal, représentations et discours : contrôle, légitime défense et amour}

\section{Sylvie Frigon}

Volume 29, numéro 2, automne 1996

L'homicide conjugal au Canada

URI : https://id.erudit.org/iderudit/017386ar

DOI : https://doi.org/10.7202/017386ar

Aller au sommaire du numéro

Éditeur(s)

Les Presses de l'Université de Montréal

ISSN

0316-0041 (imprimé)

1492-1367 (numérique)

Découvrir la revue

Citer ce document

Frigon, S. (1996). Éditorial : homicide conjugal, représentations et discours : contrôle, légitime défense et amour. Criminologie, 29(2), 3-9.

https://doi.org/10.7202/017386ar d'utilisation que vous pouvez consulter en ligne.

https://apropos.erudit.org/fr/usagers/politique-dutilisation/ 
Aujourd'hui, on ne peut penser et théoriser l'homicide conjugal sans interroger les rapports hommes-femmes tant au niveau des rapports humains que de la réaction sociale ${ }^{2}$. Dans ce numéro thématique, nous examinerons l'homicide conjugal commis par les hommes (uxoricide ou fémicide conjugal) et celui commis par les femmes («maricide» ou homicide conjugal féminin). Deux articles porteront sur le premier thème et trois sur le second.

Le Larousse définit l'uxoricide comme le meurtre de l'épouse par le mari et vient du latin uxor, -oris, épouse; 1531. Certains préfèrent parler de fémicide conjugal pour ne pas dissimuler que ce sont des femmes qui sont tuées. Par ailleurs, il est intéressant de constater qu'aucun terme spécifique, à notre connaissance, n'est utilisé pour référer au meurtre de l'époux par la femme. Nous parlerons ici de «maricide" ou d'homicide conjugal féminin.

Le fémicide conjugal est beaucoup plus fréquent que le maricide (Wilson et Daly, 1994). En effet, au Canada, entre les années 1961 et 1990, 2129 conjointes ont été tuées par leur conjoint et 782 conjoints ont été tués par leur conjointe (Silverman et Kennedy, 1993 :70). Les données du Centre canadien de la statistique juridique indiquent que $14 \%$ des homicides conjugaux commis entre 1974 et 1986 le sont par des femmes (Côté, 1991). De plus, il semble que les raisons qui motivent le passage à l'acte varient selon le sexe. D'après les recherches, un très grand nombre d'hommes qui tuent leur conjointe le font par stratégie d'appropriation, la séparation ou la menace de séparation de la part de la femme augmentant le risque d'uxoricide. Par ailleurs, il semble que pour les femmes, le geste meurtrier soit perçu, la plupart du temps, comme une stratégie de protection ou d'auto-préservation, car elles se voyaient dans une situation de "légitime défense». Une phrase, qui revient souvent dans le discours des femmes ${ }^{3}$, pourrait résumer une des raisons les plus importantes qui poussent les

1. L'auteure est professeure adjointe au département de criminologie, à l'Université d'Ottawa.

2. Ce numéro s'interroge sur l'homicide conjugal dans les relations hétérosexuelles mais, bien que rare, le meurtre de conjoints de même sexe existe et nécessiterait une analyse particulière.

3. Propos recueillis dans notre recherche archivique (Frigon, 1995 et ce numéro). et entrevues réalisées avec des femmes « maricides » condamnées au Canada, en France et en Belgique. 
femmes à tuer un conjoint: "C'était ma vie ou la sienne $\$ 4$ *Pour les hommes, ce serait plutôt: "Si je ne peux t'avoir, personne ne t'aura (voir Wilson et Daly; Boisvert et Côté, ce numéro). En effet, selon Boisvert, on pourrait résumer cela en une proposition : «Dans les homicides commis au sein d'une relation de couple, les hommes tuent parce qu'ils refusent que leur fermme leur échappe alors que les femmes tuent pour échapper à leur conjoint. "

L'uxoricide a souvent été présenté comme un «crime passionnel». Toute une mythologie romantique a contribué à cette représentation, que ce soit dans la littérature, les médias ou le cinéma. Cette mythologie se perpétue dans le droit. En effet, le droit présente les hommes comme ayant perdu la maîtrise d'eux-mêmes, ayant été «provoqués», ayant été la proie de leurs "passions », etc. Par exemple, la défense de provocation énoncée à l'article 232 du Code criminel canadien enchâsse juridiquement cette vision. Par ailleurs, cette position a été réfutée par une kyrielle d'auteurs (voir Boisvert et Côté, ce numéro). Ainsi, la violence mortelle et non mortelle contre les femmes n'est pas une perte mais plutôt une stratégie de contrôle.

Le maricide, de son côté, a peu été exploré. Mais déjà, historiquement, il était puni plus sévèrement (crime de «petite trahison») étant perçu comme le "crime du siècle", le geste d'une femme dépravée et le pire crime, comme en témoignent ces quelques citations. Dans l'adresse du juge au jury dans la cause de Tommasina Teolis en 1935 à Montréal, on peut lire: "Nous sommes en présence d'un meurtre odieux, peut-être le plus brutal que nos annales judiciaires aient enregistré ». Ou bien encore dans la plaidoirie de la Couronne dans la cause de Cordélia Viau en 1898: "Il s'agit du crime le plus abominable qui soit relaté dans nos annales judiciaires..." Et pour finir, dans l'adresse du juge au jury dans la cause de Marie Beaulne en 1929: «... C'est l'acte de trahison le plus complet qu'on puisse commettre..." (voir Bernier et Cellard, ce numéro). Ou bien, plus près de nous, les propos du juge Bienvenue en 1995 dans la cause de Mme Tracy Théberge, une femme de 35 ans condamnée pour Je meurtre de son conjoint :

L'on dit avec raison, et depuis toujours, que lorsque la femme, qui a toujours été à mes yeux l'être le plus noble de la création, [...] elle s'élève plus haut que l'homme, [...] Mais l'on dit aussi, et cela aussi je le crois, que lorsqu' elle

4. Notons, à titre d'exemples, le procès de Mme Frances Harrop en 1940 à Winnipeg, pendant lequel on peut lire dans le rappont de police : «It was a case of him taking my life or me taking his" (Frigon, 1995). Et dans la cause d'Angélique Lyn Lavallée, on peut lire dans le témoignage de l'accusée sur ce que la victime lui a dit: «Ou tu me tues, ou moi je t'aurai.» (R. C. Lavallée, [1990], p. 856). Plusieurs autres exemples pourraient être donnés. 
décide de s'abaisser, la femme le fait, hélas jusqu'à un niveau de bassesse que l'homme le plus vil ne saurait lui-même atteindre ${ }^{5}$.

Nous trouvons un écho à ces propos chez le célèbre Lombroso plus de 100 ans auparavant :

[...] il est évident que de la demi-criminaloide inoffensive qu'est la femme nornale surgira une criminelle-née plus terrible que n'importe quel homme criminel $[. .$.

En outre, Ja criminelle-née est pour ainsi dire une exception à double titre, comme criminelle et comme femme [...] Elle doit donc, comme double exception, être plus monstrueuse (Lombroso, 1895: 360-361 ; je souligne).

Le maricide êtait aussi perçu comme une transgression au rôle de femme, d'épouse, de mère, et comme une révolte inacceptable contre l'autorité masculine. Ainsi, lorsque les femmes résistaient à la violence du conjoint et dans certains cas avaient recours à l'homicide, la violence conjugale n'avait que très peu de place lors des procès. Il faudra attendre la décision Lavallée de la Cour suprême du Canada en 1990 pour que la violence conjugale ait un rôle à jouer dans les décisions ${ }^{6}$. Cela sera présenté sous la forme du «syndrome de la femme battue » (SFB).

Ainsi, comme nous le verrons dans ce numéro spécial sur l'homicide conjugal, les hommes sont souvent «excusés» ou ils voient leur responsabilité atténuée par la justice, tandis que les femmes ne peuvent bénéficier des défenses comme la provocation ou la légitime défense telles qu'établies dans le code criminel puisqu'elles ne peuvent tenir compte de leurs expériences, d'où la nécessité d'avoir recours au SFB. Or, les femmes sont à l'extérieur de la logique pénale, tandis que les hommes homicides correspondent aux critères jurisprudentiels établis ${ }^{7}$.

5. La Presse, 9 décembre 1995 ; je souligne.

6. La violence conjugale dans les cas de maricide a aussi été mise en évidence dans différentes productions cinématographiques, littéraires et thêtrales. Notons, par exemple, le documentaire When Women Kill (1994), produit par l'Office national du film, qui raconte l'histoire de quatre femmes au Canada ayant tué leur conjoint après des annees de violence conjugale. $L$ 'histoire de Jane Stafford, une femme battue qui a tué son conjoint, a aussi été relatée dans un livre intitulé Life with Billy, qui a été porté à l'écran en 1993. Même le grand écrivain de suspense américain, Stephen King, a examiné le maricide dans Dolores Clairborne (1993) également porté à l'écran. La dramaturge terre-neuvienne Berni Stapleton a, pour sa part, créé une pièce de théâtre intitulée Offensive to Some (1996), qui se déroule dans la cellule d'une femme emprisonnée pour le meurtre de son conjoint violent.

7. Au Canada à la suite de la décision Lavallée et à la demande de la société Elizabeth Fry, une révision (en bloc) des dossiers des femmes condamnées pour homicide commis dans le contexte d'une relation empreinte de violence est présentement 
Dans la première partie de ce numéro, le premier article, celui de Frigon, est une réflexion sur les femmes «maricides» où l'auteure tente de retracer l'émergence de la reconnaissance sociale et légale de la violence conjugale dans ces procès. La décision de la Cour suprême du Canada en 1990 dans la cause Lavallée sert de pivot à l'analyse, car une rupture épistémologique importante s'annonce dans cette décision. Un traitement d'ensemble est proposé ici dans le but de mieux comprendre et situer la portée de cette question et le contexte dans lequel elle s'inscrit. Ce questionnement permet de cerner le passage de la non-reconnaissance à la reconnaissance de la violence conjugale dans le traitement judiciaire des femmes battues qui ont tué leur conjoint, et de mettre en évidence l'absence de la perspective des femmes. Qui plus est, cette façon d'aborder la problématique nous permet de mieux comprendre les décisions légales, car il apparaît essentiel pour l'auteure de situer la problématique de l'homicide conjugal dans son ensemble, afin d'amorcer quelques réflexions et d'envisager certaines stratégies futures. Finalement, il est possible de mieux comprendre le contexte d'émergence de la preuve d'expert du «syndrome de la femme battue» (SFB) et la façon dont la perspective des femmes a été construite et mise en valeur dans la décision Lavallée en 1990, avec les limites qui y sont inhérentes.

Jusqu'à très récemment, la perspective des femmes était évacuée lors de ces procès. Dans cet ordre d'idée, Bernjer et Cellard, dans leur article, nous convient à un voyage au Québec, de la fin du XIX $X^{e}$ siècle jusqu'à la Deuxième Guerre mondiale, en mettant en relief les procès des maricides et les représentations féminines qui y sont (re)produites. Leur recherche archivique montre qu'entre 1898 et 1940, au Québec, cinq femmes furent condamnées à la peine de mort pour le meurtre de leur conjoint. Le but poursuivi dans cette recherche n'était pas, au dire des auteurs, « de savoir si ces personnes [femmes] étaient coupables, mais plutôt si, en raison du contexte dans lequel le geste qu'on leur reprochait fut jugé, elles eurent droit à un procès juste et équitable ". Qui plus est, les femmes «maricides" étaient une "dérogation plus patente au rôle traditionnel qui était alors assigné à la femme dans la société canadienne-française» : une transgression à leur rôle de femme, d'épouse et de mère. La femme "maricide " ne sera-t-elle pas représentée par les «définisseurs de normes» (Lévesque, 1989) comme «la femme qui, en assassinant ou en participant au meurtre de son époux, menaçait les fondements mêmes de la société »?

menée par la juge Lynn Ratushny à la demande du ministère de la Justice en octobre 1995. Quatre-vingt-seize demandes de révisions ont été présentées par des femmes dont la majorité avaient tué un conjoint. Près d'une vingtaine de demandes ont été déposées par des femmes autochtones. 
Dans la deuxième partie, trois articles portent sur l'uxoricide ou fémicide conjugal. Basé sur une approche épidémiologique, l'article de Wilson et Daly porte sur les liens psychologiques entre la possessivité sexuelle masculine et la violence contre les femmes, la relation entre certains marqueurs démographiques et les tendances quant aux risques d'assauts mortels et non mortels contre les femmes. Après plus de quinze années de recherche dans le domaine, les auteurs considèrent l'uxorìcide comme un "épiphénomène des tendances masculines à la coercition physique et mentale" et a des points de convergence avec la violence non mortelle contre les femmes. En effet, selon les auteurs, "l'uxoricide est, la plupart du temps, la pointe de l'iceberg masquant la violence non mortelle des conjoints contre leur femme ". En effet, des profils parallèles quant au type d'union (risque plus élevé pour les unions libres que pour les mariages) et de l'âge (les jeunes épouses seraient plus menacées que les épouses plus âgées) émergent. Néanmoins, le Québec et le Nouveau-Brunswick diffèrent du reste du Canada sur certains aspects.

Boisvert, pour sa part, rapporte les résultats d'une étude comparative entre deux périodes, soit de 1954 à 1962 et de 1985 à 1989 , sur l'homicide conjugal des hommes à Montréal. Les raisons qui semblent motiver le passage à l'acte sont la possessivité, la jalousie masculine, la réaction à la dépossession et la domination. De plus, les protagonistes des homicides conjugaux pendant ces deux périodes viennent de milieux socio-économiques et socio-éducationnels peu favorisés. Les données de cette recherche indiquent également que $30 \%$ des femmes tuées par leur conjoint avaient été victimes de violence conjugale signalée à la police. De plus, la séparation n'était pas toujours une façon efficace de se soustraire à la violence meurtrière car, selon l'auteure, sur 66 affaires d'homicide conjugal, on comptait 15 relations de couple rompues et 12 où il était question de séparation.

Boisvert s'interroge aussj sur deux déclencheurs individuels, la « folie " et la consommation d'alcool, conditions qui ont souvent été identifiées comme propices à l'explosion de la violence. L'auteure relève que seulement $12 \%$ ( 9 sur 77 ) des hommes ont été acquittés en raison d'aliénation mentale, ce qui l'amène à suggérer que « [c]e pourentage de $12 \%$ n'est pas négligeable mais on voit qu'il serait inadéquat d'interpréter toute la violence meurtrière exercée contre les femmes comme étant le produit de comportements désorganisés ou pathologiques [...] La règle, c'est plutôt que l'agresseur est en bonne santé mentale et responsable». Ces données empiriques ne permettent pas non plus d'identifier la consommation d'alcool comme facteur déterminant. Il faudrait plutôt parler de co-incidence puisque 19 hommes sur un total de 77 étaient sous l'effet de l'alcool $(25 \%)$. Ainsi, Boisvert recommande de «dépasser les gestes individuels posés par tel individu en particulier et de se placer sur le palier macrosocio- 
criminologique, soit d'interpréter cette violence en examinant les éléments de l'organisation sociale» et de comprendre les uxoricides comme «une des manifestations d'une société où les hommes dominent les femmes ».

Pour clore la partie thématique de ce numéro, Côté analyse le discours juridique et judiciaire de la jurisprudence entourant la défense de provocation et le fémicide conjugal. Selon l'auteure, le droit "excuse » la violence des hommes contre les femmes en s'appuyant sur des valeurs patriarcales et des techniques d'interprétation judiciaire. En citant de multiples décisions, allant jusqu'à la décision Thibert de la Cour suprême du Canada en janvier 1996, Côté soutient que le droit établit clairement que «le fait de porter atteinte au droit d'un homme de s'approprier une femme, de la contrôler et d'avoir un accès sexuel exclusif à sa personne demeure de la "provocation" au regard du droit». Cette «interprétation patriarcale", toujours selon Côté, est «fondée sur la reconnaissance historique des droits de propriété du mari sur son épouse, de son droit de contrôler "sa" femme et de se venger de toute atteinte à son droit de jouir en exclusivité de sa sexualité...". Cette interprétation juridique de la provocation n'est possible que si l'on fait abstraction du contexte dans lequel l'homicide a eu lieu.

Il est par ailleurs intéressant de constater que la défense de provocation repose sur l'idée que la personne a perdu le contrôle et n'a pu maîtriser sa colère; mais cette idée de perte de contrôle, comme le souligne Côté, a sérieusement été remise en question. En effet, les études sur la violence conjugale «indiquent que les hommes violents utilisent la violence physique pour contrôler le comportement de leur conjointe ou pour prévenir une perte de contrôle..." ". Ainsi, la violence mortelle et non mortelle contre les femmes n'est pas une perte de contrôle mais plutôt une stratégie de contrôle. À la Jumière de ces données et à l'instar d'autres auteurs, Côté recommande donc d'abolir la défense de provocation ou au moins de l'in. terdire dans les causes de fémicide conjugal.

Ainsi, les articles de ce numéro montrent éloquemment comment les représentations populaires et sociales, tout comme le traitement juridique et judiciaire de l'homicide conjugal, reconduisent et renvoient aux rapports sociaux de sexe inégalitaires.

Dans le présent numéro de la revue Criminologie, outre des articles thématiques, on trouve deux autres articles. Le premier, de Facy, Brochu et Simon, présente une comparaison des systèmes français et québécois en matière de traitement judiciaire réservé aux toxicomanes. Les auteurs montrent qu'au Canada, le recours à l'incarcération porte à réflexion, tandis que l'injonction thérapeutique en vigueur en France soulève d'autres questions. Gorgeon, pour sa part, présente les résultats de plusieurs recherches sur la socialisation professionnelle des policiers. Les études montrent que les 
caractéristiques culturelles spécifiques existantes dans la profession exercent une influence sur les nouvelles recrues.

\section{BIBLIOGRAPHIE}

CÔTÉ, A. (1991), La Rage au Caur : rapport de recherche sur le traitement judiciaire de lhomicide conjugal au Québec, Regroupement des femmes de la Côte-Nord.

FRJGON, S. (1995), "When Women Kill Violent Husbands in Canada, 1871-1946: Drama, Disqualification of Women's Voices, Resistance and Male Tyranny ", Conférence présentée à la British Criminology Conference, à l'Université de Loughborough en Angleterre du 18 au 21 juillet 1995.

LÉVESQUE. A. (1989), La norme et les déviantes, des femmes au Québec pendant l'entre-deux-guerres, Montréal, Les éditions du Remue-Ménage.

LOMBROSO, C. et FERRERO, G. (1991; $1^{\text {re }}$ éd. : 1895), La femme criminelle et la prosituée, Grenoble, Éditions Jérôme Millon.

R. c. Lavallée (1990), 76 C.R. (3rd) 329 (S.C.C.).

SILVERMAN, R. et KENNEDY, L. (1993), Deadly Deeds : Murder in Canada, Scarborough, Nelson Canada.

WILSON, M. et DALY, M. (1994), "Les homicides entre les conjoints", Juristat, Centre canadien de la statistique juridique, Ministère de l'Industrie, des Sciences et de la Technologie, vol. $14, n^{\circ} 8$. 